\title{
Rigaku's SmartLab XRD tool: All cutting-edge technologies in one instrument.
}

\author{
Artem Shalimov \\ Rigaku Europe SE, Neu-Isenburg, Germany; \\ artem.shalimov@rigaku.com
}

The first generation of the SmartLab XRD multipurpose diffractometer was launched in 2008. It involved immense effort from Rigaku engineers, X-ray scientists and application experts aiming to deliver a multifunctional XRD instrument to cover wide range of X-ray scattering techniques in the lab environment. 10 years later, after the success of the first SmartLab, Rigaku released the new generation of SmartLab, implementing newest solutions and technologies based on scientific and industrial demands fulfilling users' needs and requests. This overview presentation provides an update of the new solutions implemented in the second generation of SmartLab resulting in substantial extension of applicability of the instrument.

In addition to standard Powder and Thin Film XRD applications, the new SmartLab has been updated with a new family of Cross Beam Optics (CBO), Goebel's mirror equivalent, which includes elliptical mirror CBO-E, and flat mirror CBO- $\alpha$ for different wavelengths including $\mathrm{Cu}, \mathrm{Mo}$, and $\mathrm{Ag}$. In addition, an X-ray polycapillary unit $\mathrm{CBO}-\mathrm{f}$ and confocal mirror set $\mathrm{CBO}-\mu$ has been designed for micro-area testing utilizing focused beams of $400 \mu \mathrm{m}$ and $50 \mu \mathrm{m}$ respectively.

Furthermore, newly designed sample attachments along with the appropriate optical set, enable uncompromised SAXS, WAXS, GISAXS and GIWAXS measurements that require utilization the large 2D acquisition area. The requirement of 2D data collection over large angular space is fulfilled with in-house development and manufacturing of HyPix400 and HyPix3000 2D detectors characterized by outstanding dynamic range ( $>2 \mathrm{Mcps} / \mathrm{pixel})$, read out speed (zero dead-time) and robustness. Due to their unique technology HyPix detectors do not require primary X-ray beam attenuation and can be safely used with strong beams, including exposure to the direct beam from a $9 \mathrm{~kW}$ X-ray source.

The Differential Scanning Calorimetry (DSC) and reaction sample chamber (Reactor X) for in-situ studies is of particular interest to research scientists. This instrument set-up is ideal for examining phase transitions under alternating temperature and humidity as the results can be directly observed when combining XRD and DSC in one experiment. Materials transformations in an atmosphere of reactive gases (mixtures of gases, or also in air or vacuum) may be studied using Reactor $\mathrm{X}$ which is capable to elevate temperature up to $1000^{\circ} \mathrm{C}$ with ultra-rapid ramp rate. Additionally, following high demand in investigations of energy storage materials, Rigaku has designed a set of battery attachments for reflection and transmission geometries for in-operando experiments.

New optics, sample attachments and developments in detector technology have enabled the SmartLab to achieve best-in-class experiments for Powder XRD and Thin Film structural analysis, phase transitions, Small- and Wide-Angle X-ray Scattering, PDF, micro-area testing, pharmaceutical research, applications for steels, alloys, and multifunctional materials.

Keywords: XRD, Thin Films, PDF, SAXS, WAXS 